\title{
Cannabis and intractable chronic pain: an explorative retrospective analysis of Italian cohort of $6 / 4$ patients
}

This article was published in the following Dove Press journal:

Journal of Pain Research

22 May 2017

Number of times this article has been viewed

\author{
Guido Fanelli, ${ }^{1,2}$ \\ Giuliano De Carolis ${ }^{3}$ \\ Claudio Leonardi ${ }^{4}$ \\ Adele Longobardi ${ }^{5,6}$ \\ Ennio Sarli ${ }^{7,8}$ \\ Massimo Allegri ${ }^{1,2}$ \\ Michael E Schatman ${ }^{9}$
}

'Anesthesia, Critical Care and Pain Medicine Unit, Division of Surgical Sciences, Department of Medicine and Surgery, University of Parma, ${ }^{2}$ Anesthesia, Intensive Care and Pain Therapy Service, Azienda Ospedaliero Universitaria Parma, Parma, ${ }^{3}$ Pain Therapy Service, Azienda Ospedaliero Universitaria Pisana, Pisa, ${ }^{4}$ Department of Drug Addiction Diseases, Local Public Health of Rome, Rome, ${ }^{5}$ Department of Neurosciences, Reproductive and Odontostomatological Sciences, University of Naples "Federico II", Naples, 'Young Against the Pain (YAP) Group, Parma, ${ }^{7}$ Progetti Live Surgery, ${ }^{8}$ PinHub Group, Florence, Italy; ${ }^{9}$ Department of Public Health and Community Medicine, Tufts University School of Medicine, Boston, MA, USA

Correspondence: Guido Fanelli Department of Medicine and Surgery, University of Parma, Via Gramsci, 43। 26 Parma, Italy

Tel +3905 21703965

Email guido.fanelli@unipr.it
Background: Despite growing interest in the therapeutic use of cannabis to manage chronic pain, only limited data that address these issues are available. In recent years, a number of nations have introduced specific laws to allow patients to use cannabis preparations to treat a variety of medical conditions. In 2015, the Italian government authorized the use of cannabis to treat several diseases, including chronic pain generally, spasticity in multiple sclerosis, cachexia and anorexia among AIDS and cancer patients, glaucoma, Tourette syndrome, and certain types of epilepsy. We present the first snapshot of the Italian experience with cannabis use for chronic pain over the initial year of its use.

Methods: This is a retrospective case series analysis of all chronic pain patients treated with oral or vaporized cannabis in six hubs during the initial year following the approval of the new Italian law (December 2015 to November 2016). We evaluated routes of administration, types of cannabis products utilized, dosing, and effectiveness and safety of the treatment.

Results: As only one of the six centers has extensively used cannabinoids for intractable chronic pain (614 patients of 659), only the population from Azienda Ospedaliero Universitaria Pisana (Pisa) was considered. Cannabis tea was the primary mode of delivery, and in almost all cases, it was used in association with all the other pain treatments. Initial and follow-up cannabinoid concentrations were found to vary considerably. At initial follow-up, $76.2 \%$ of patients continued the treatment, and $<15 \%$ stopped the treatment due to side effects (none of which were severe). Conclusion: We present the first analysis of Italian clinical practice of the use of cannabinoids for a large variety of chronic pain syndromes. From this initial snapshot, we determined that the treatment seems to be effective and safe, although more data and subsequent trials are needed to better investigate its ideal clinical indication.

Keywords: cannabis, cannabinoids, chronic pain, safety, cannabidiol

\section{Background}

The past years have witnessed a growing interest in the therapeutic use of cannabis and its constituents to manage chronic pain. ${ }^{1}$ Irrespective of the number of new trials examining the use of cannabinoids for chronic pain, the evidence of its effectiveness and its safety remains limited. ${ }^{2}$ The pharmacology of Cannabis sativa (the strain generally used to treat chronic pain) is quite complex, as it contains $\sim 100$ distinct cannabinoids, ${ }^{3}$ the relative levels of which largely determine their therapeutic effect. ${ }^{4}$ Of the numerous cannabinoids, $\Delta 9$-tetrahydrocannabinol $(\Delta 9$-THC) is considered the most psychoactive. ${ }^{5,6}$ The current definition of cannabinoids includes all endogenous and exogenous compounds that act on cannabinoid receptors. ${ }^{7}$ 
THC is not the only cannabinoid with therapeutic effects $^{8}$, as cannabidiol (CBD) is used in the treatment of several different conditions. CBD is important not only for its therapeutic effects but also for its ability to mitigate euphoria and other side effects caused by THC, ${ }^{9}$ thereby increasing the potential therapeutic applications of cannabis. A lack of standardized dosing and uncertainty regarding the ideal ratio between $\mathrm{THC}$ and $\mathrm{CBD}$ continue to limit the medicinal usage of marijuana. ${ }^{10} \mathrm{~A}$ review by Koppel et $\mathrm{a}^{11}$ analyzed six different neurological disease states and symptoms and a variety of THC/CBD ratios and methods of administration (e.g., oral, oromucosal, and inhaled) without obtaining clear evidence for specific indications. As there are numerous products containing myriad THC/CBD ratios, it remains extremely challenging to draw reproducible data regarding their effectiveness in the treatment of pain. ${ }^{10}$

There are several methods of administration of cannabinoids including smoking, orally (by infusion or by extraction in oil, as well as through edible products), vaporizing, and transdermally. While smoking remains the most common form of administration, a recent study found that in jurisdictions in which medical marijuana is legal, smoking is not necessarily the preferred route of administration. ${ }^{12}$ However, variance in routes of administration makes comparison of results of different studies questionable. As a number of different compounds and methods of administration exist, thorough education of the physician (as well as the patient) is imperative prior to prescribing or recommending these compounds. ${ }^{13}$

The most comprehensive meta-analysis of inhaled cannabis for chronic pain to date ${ }^{14}$ has recently supported its short-term effectiveness in treating neuropathic pain. The authors also concluded that more studies are needed in order to evaluate long-term effectiveness and safety. These conclusions are consistent with those from Schatman's 2015 comprehensive review. ${ }^{10}$ Regarding safety, Aggarwal ${ }^{15}$ opined that although "little data are available on the risks associated with long-term medical use in published clinical trials," it can still be used to treat complex chronic pain conditions. More recently, Ware et a ${ }^{16}$ published a 1 -year follow-up trial in order to better investigate the long-term efficacy and safety. The median dose of cannabis was $2.5 \mathrm{~g}$ daily. The authors did not find differences in serious adverse events between chronic pain patients treated with or without cannabis. Nevertheless, a higher incidence of mild adverse effects was registered among patients treated with cannabis.
Even though the evidence is still weak and more studies are needed, there is significantly increasing availability of cannabis to treat chronic pain in several different countries, especially in the US and Canada. ${ }^{17}$ Understanding of complex policy and public health issues is imperative in order to fully understand the distinction between medical vs. recreational utilization of cannabis. Savage et al recently concluded that there is a need not only for additional empirical investigation but also for increased research funding to help us develop a better understanding of how to make cannabinoids more effective and safer. ${ }^{17}$

Questions have also been raised regarding the legitimacy of dispensaries' clientele. ${ }^{10}$ It has been demonstrated that most dispensary customers had initiated marijuana use in adolescence, with one-half presenting with indications of risky alcohol use and $20 \%$ presenting with recent histories of prescription medication or illicit drug abuse..$^{15}$

Hence, many concerns are arising regarding the distinct possibility that in the US and Canada, some of the same problems that these nations have had with prescription opioid abuse will potentially develop in relationship to "medical" marijuana. Currently, the US is aggressively fighting its opioid crisis while simultaneously liberalizing access to cannabis - for both medical and recreational utilization. ${ }^{18}$

An Italian law approved in $2015^{19}$ authorizes the use of cannabis to treat chronic pain. The law allows for the utilization of cannabis not only for neuropathic pain but also for all chronic pain conditions, as well as for spasticity, cachexia, and anorexia among AIDS and cancer patients, ocular hypertension in glaucoma, the alleviation of spasms in Tourette syndrome, and some types of epilepsy, reiterating that cannabis-based drugs should be prescribed only "when other available medications have proven to be ineffective or inadequate to the therapeutic needs of the patient."

In order to reduce the costs of cannabinoid products, the Italian government in 2014 committed its Military ChemicalPharmaceutical Factory to cultivate cannabis to be distributed to all pharmacies across the country (initial production is scheduled for early in 2017). In the meantime, physicians can legally prescribe different cannabis products (such as Bedrocan, Bediol, and Bedrolite), with different THC and CBD concentrations; these drugs can be administered orally (e.g., through infusions in olive oil) or via inhalation. Bedrocan's constituents are $22 \% \mathrm{THC}$ and $<1 \% \mathrm{CBD}$, while Bediol contains $6.5 \% \mathrm{THC}$ and $8 \% \mathrm{CBD}$ and Bedrolite contains $9 \% \mathrm{CBD}$ and $0.4 \% \mathrm{THC}$ and is accordingly considered "non-psychoactive." 20

All patients treated with cannabinoids have to be registered through a Ministry of Health database. In recent months, 
the society that includes all second-level (hubs) pain centers (PinHub, www.pinhub.it) has received a directive to evaluate all of these data among its centers.

This paper presents a retrospective analysis of a case series of all chronic pain patients treated in one PinHub center over the past year in order to provide a snapshot of the initial Italian experience with legalized cannabis use for chronic pain. The end point of this study has been the evaluation and identification of clinical indications and dosages currently used in second-level center of pain therapy. Although our intention was to include data from all six of the centers initially involved in the study, a paucity of data from five of the six centers precluded doing so.

\section{Methods}

Following the approval of the Italian law sanctioning the medical use of cannabis (2015), all patients who initiated the use of medical cannabis have had to be registered, with their consent, in a database to evaluate specific data (Figure 1). A retrospective group analysis of a case series of all chronic pain patients treated in one of the second-level pain clinics affiliated with the PinHub group in the first year following the approval of the Italian law (December 2015 to November 2016) was performed. The clinical centers intended to be involved in this study were the pain therapy services of the following hospitals: SS Antonio e Biagio Hospital (Alessandria), SS Annunziata Hospital (Chieti), Monaldi Hospital (Naples), Verona University Hospital (Verona), Siena University Hospital (Siena), and Azienda Ospedaliera Universitaria Pisana (Pisa).

Patients gave their permission to use their data when the physician filled out the case report form (CRF). This research did not require approval by the Institutional Review Boards of the aforementioned clinical centers as we used only raw data that was completely de-identified and anonymous. The data were presented in accordance with the Strengthening the Reporting of Observational Studies in Epidemiology (STROBE) guidelines. ${ }^{21}$

This study evaluated all patients registered as they had initiated treatment with cannabinoids for chronic pain based upon the judgment of their pain therapists. In accordance with the Italian law, all patients treated with cannabinoids have had to be $\geq 18$ years of age and determined to suffer from refractory chronic pain. According to Italian law, cannabis could be prescribed with only two routes of administration: orally (infusion or extraction in olive oil) or through inhalation. Smoking the cannabis is not permitted, so vaporization was the technique used for inhalation. Currently, there are no national guidelines to be followed by physicians.
Furthermore, comorbidities and their severity that might preclude the treatment are not commonly predefined among the centers that are using this treatment.

The primary end point of this study was to provide insight into how the Italian second-level pain centers are utilizing medical cannabinoids in terms of routes of administration. Secondary end points included the determination of the types of cannabis products most commonly utilized, as well as dosing.

Participating physicians were able to choose among infusion or inhalation via vaporization of the following types of cannabinoid products: pure Bediol, Bediol combined with Bedrocan, pure Bedrocan and Bedrolite, and oil infused with Bedrocan. As $\sim 92 \%$ of the patients in the study used the highTHC, low-CBD Bedrocan, it was unable to assess differences in efficacy and adverse events between the strains utilized in this preliminary investigation (Figure 1).

Furthermore, the study evaluated effectiveness and safety through the analysis of patients who had at least one follow-up subsequent to initial prescription based on the data provided by the CRFs defined by the Italian Health Ministry.

Through the data requested by the Italian Health Ministry for follow-up purposes, the study evaluated the dosages utilized, whether the therapy has been continued, clinical efficacy (determined by patient-reported levels of pain severity), and the reason for discontinuing treatment (pain worsened, pain not clinically improved, or presence of intolerable side effects). Although assessing other outcomes such as functionality would have been useful, the Italian Health Ministry has requested the provision only of data pertaining to pain relief.

Data have been obtained by the CRF requested by the Italian Health Ministry that has to be filled whenever patients initiated the therapy or had subsequent contact with the pain center at which they were being treated.

\section{Statistical analysis}

The sample size was not calculated a priori as this study was an explorative retrospective analysis of all patients treated with cannabinoids for a year in second-level centers in Italy after the approval of the law that legalized their use for chronic pain.

All data are presented with percentages or means, as well as standard deviations. As this initial study is a case series, it is not surprising that the data are quite heterogeneous. However, the authors chose not to formally evaluate any statistical differences between the different treatments, as there were insufficient number of subjects using any product besides Bedrocan. 


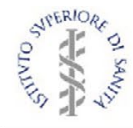

$\begin{aligned} & \text { Pilot project for Italian production of cannabis and its derivatives } \\ & \text { Case record form for cannabis-based therapies }\end{aligned}$
$\begin{array}{ll}\text { Region Hospital } & \text { Surname } \\ \text { Name } & \text { Mailing address } \\ \text { Telephone } & \square \text { Primary care physician } \\ \square \text { Hospital doctor / specialist } & \end{array}$

\section{PATIENT}

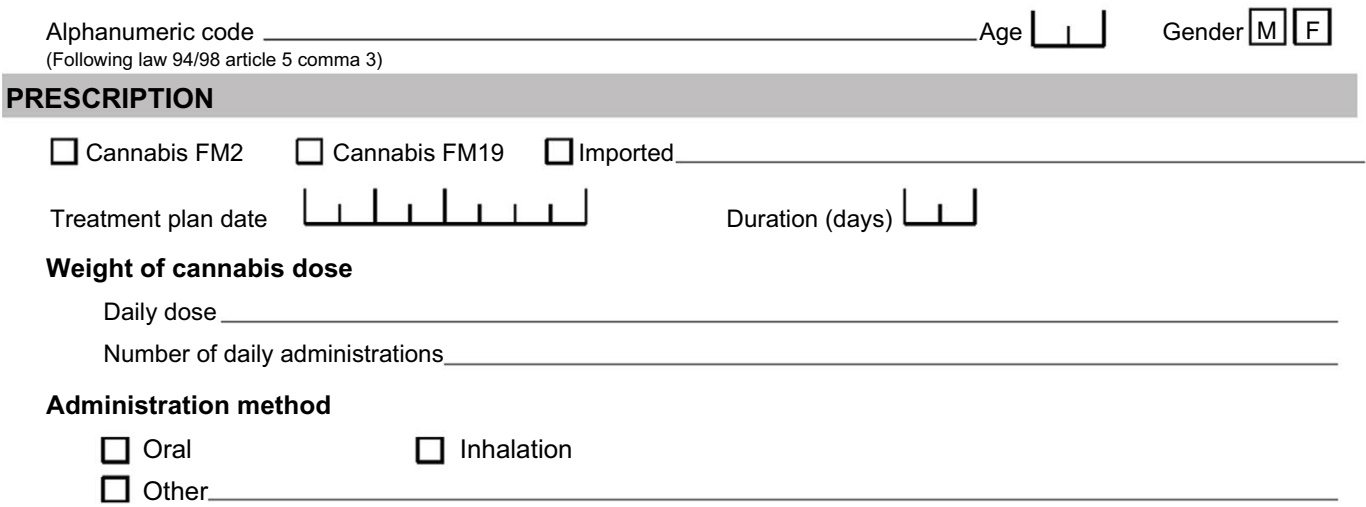

Indications for use

$\square$ Spasticity associated pain (multiple sclerosis, spinal cord injury) resistant to conventional therapies

$\square$ Neuropathic chronic pain resistant to conventional therapies

$\square$ Nausea and vomiting associated with cancer chemotherapeutic agents, radiation therapy, HIV therapy

$\square$ Cachexia, anorexia in patients with cancer, AIDS, and anorexia nervosa

$\square$ Glaucoma

$\square$ Gilles de la Tourette syndrome motor and phonic tics

$\square$ Other

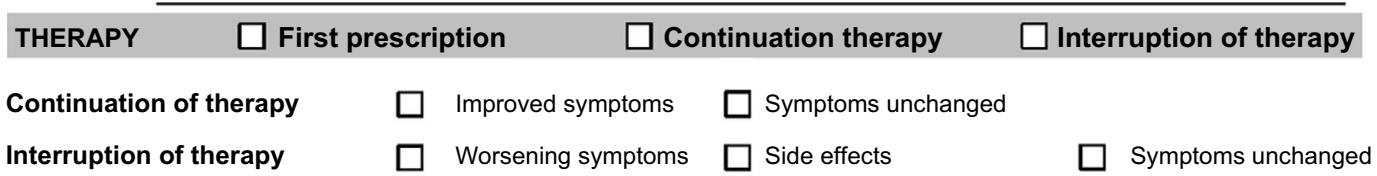

Date of interruption $\quad$\begin{tabular}{|l|l|l|l|}
\hline & & & \\
\hline
\end{tabular}
Use of medical cannabis
Replacement of conventional treaments
Integration therapy

Figure I (Continued) 


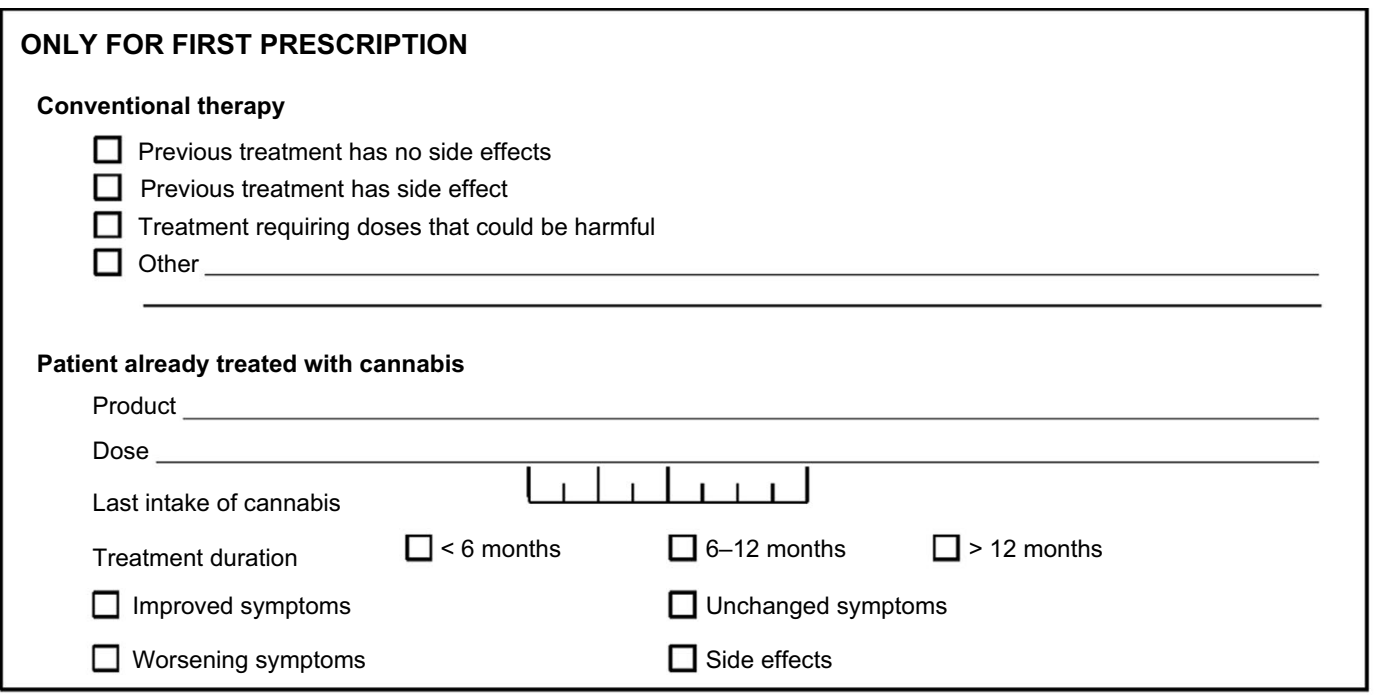

If you notice unexpected side effects, please note it

Observations

Signature

Place

Date

Figure I Case report form, approved by the Italian Health Ministry, used by clinicians who prescribed cannabis to patients.

Note: This is an English translation of the original version of the form, which was presented in Italian. FM2 and FMI9 are specific varieties of cannabis preparations made by the Italian Military Pharmacy. FM2 contains THC 6\% and cannabidiol 6-9\%; FMI 9 contains only THC 19\%.

\section{Results}

Of the 659 patients who initiated treatment with oral cannabis at all of the facilities originally intending to participate in the study, the study included only the subjects from the Azienda Ospedaliero Universitaria Pisana (Pisa) for the analysis of data. As only $6 \%$ of the patients came from the other five facilities, a comparison of inter-facility data would not have been possible. On a positive note, utilizing only the data from the Azienda Ospedaliero Universitaria Pisana also allowed for a more homogeneous evaluation of current treatment. Of all the subjects, 181 were male and 422 were female, and data

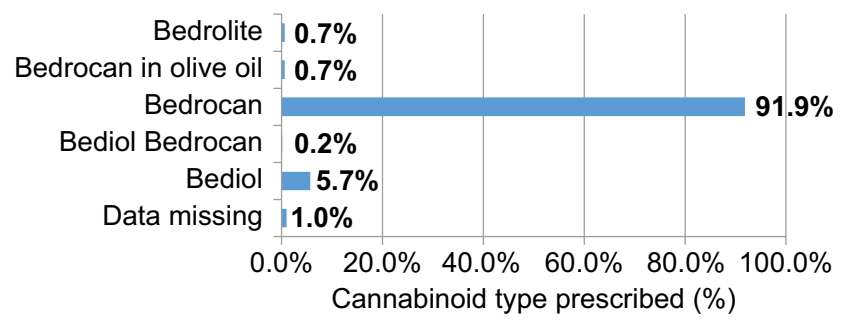

Figure 2 Type of cannabinoids prescribed.

on gender were missing for 11 subjects. The average age of subjects was $61.33( \pm 15.29)$ years. Of 614 patients whose data were used, 341 (55.5\%) had at least one follow-up at 


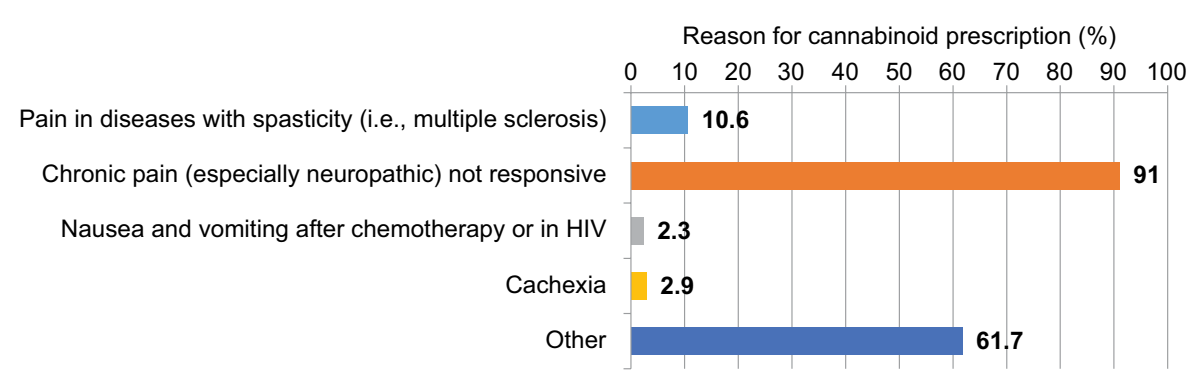

Figure 3 Reasons for which cannabinoids have been prescribed.

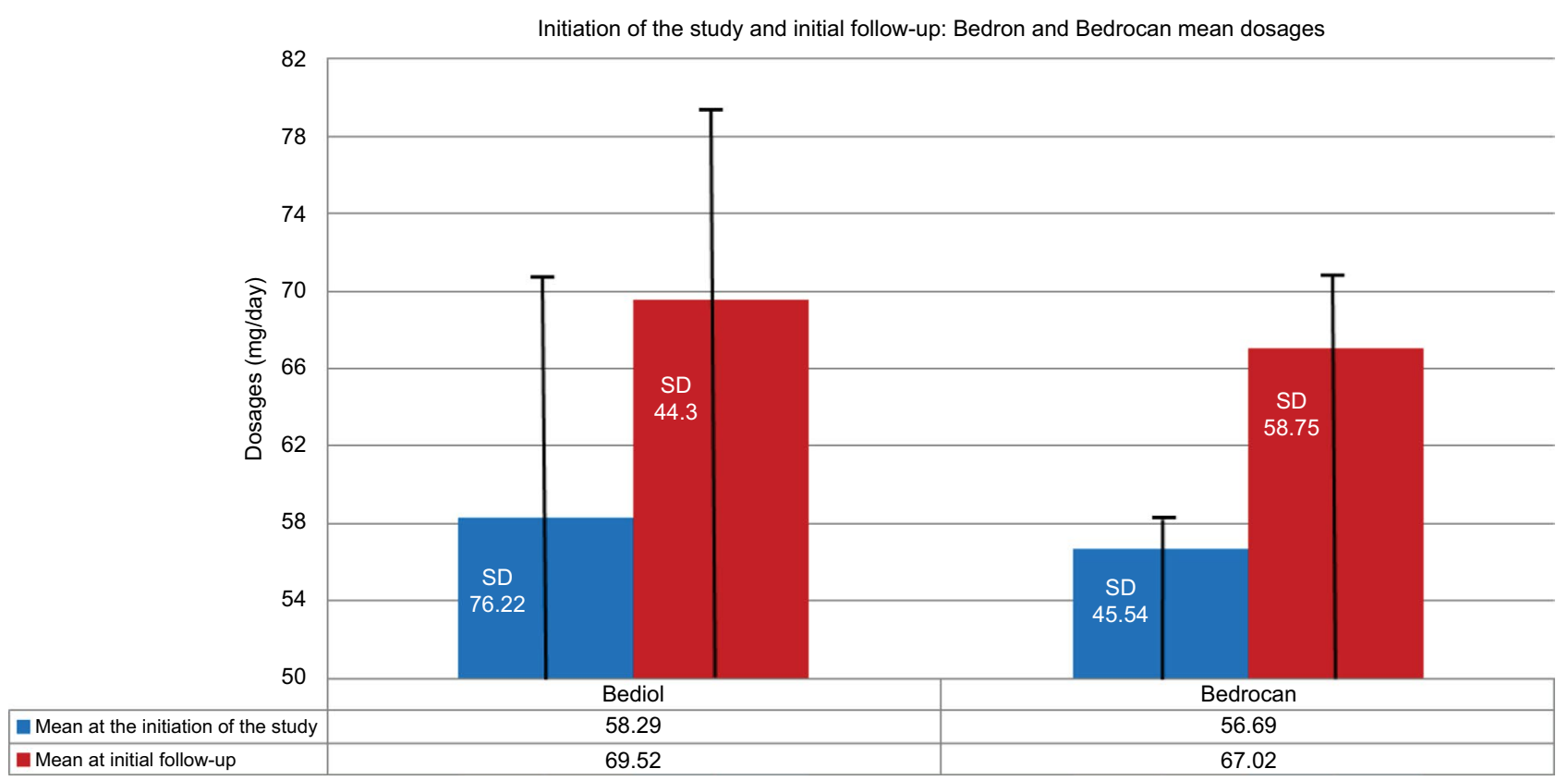

Figure 4 Mean of dosages ( $\mathrm{mg} /$ day) of Bediol and Bedrocan at the first visit and at the first follow-up.

Abbreviation: SD, standard deviation.

98.42 ( \pm 144.66$)$ days. Figure 2 illustrates the type of cannabis product used, by percentage. Figure 3 indicates the specific symptoms for which physicians initiated cannabis treatment.

In $89.2 \%$ of patients, cannabis was prescribed without discontinuing their previous analgesic therapy. At initial follow-up, $76.2 \%$ patients continued the cannabinoid therapy (of whom $64.7 \%$ reported an improvement associated with the therapy, while $34.1 \%$ reported neither an improvement nor a worsening), and $23.8 \%$ discontinued treatment $(3.7 \%$ due to a worsening of their pain, $61.7 \%$ due to side effects, $29.6 \%$ due to an unsatisfactory change of their clinical condition; for $4.9 \%$ of patients, the data were missing). There were no complaints of severe side effects, even though this may not have been directly assessed at follow-up visits. Figure 4 illustrates the dosages (mg/day) of Bedrocan and Bediol at the initiation of the study and at initial follow-up, respectively.

\section{Discussion}

Over the past year, a dramatic increase in the use of cannabis to treat chronic intractable pain has been witnessed. ${ }^{11}$
Nevertheless, specific guidelines are still missing, and there is considerable heterogeneity in the use of this drug throughout the world. In Italy, cannabis was approved legally 18 months ago for its use for several indications, including treatment of intractable pain (not specifically neuropathic pain). All patients who initiated treatment with cannabis had been required to be registered in a specific national database in order to evaluate the clinical effectiveness and safety of cannabis in the 2 years immediately following its legalization. Hence, a retrospective analysis of a case series of patients treated in a second-level pain clinic that is a part of the PinHub society was performed. This analysis attempted to provide an initial snapshot of how cannabinoids are used in Italy in order to understand how cannabis is currently used for chronic pain treatments and its implications for clinical practices.

The initial data that were obtained are those only from one of the six centers that have extensively used cannabinoids for intractable chronic pain. It was observed that cannabinoid treatment is not yet common, even though the 
new law has been approved. This situation could be compared to a similar situation observed in Italy several years ago with regard to opioids; despite the passage of legislation intended to facilitate opioid prescription, several years passed prior to the initiation of an appropriate increase in opioid prescription.

The population in this investigation that was treated was similar to that generally observed in pain clinics (average age of 61 years, and majority being female). Regarding the type of cannabinoid and modality of administration, almost all patients received infusions of Bedrocan, while administration of the extract of cannabinoids in olive oil or vaporization was quite uncommon. Hence, as these data are quite discrepant from those from Canada and the US, it is extremely difficult to compare the effectiveness and safety of the treatment to those data from studies performed in other nations. This heterogeneity currently limits the possibility of developing guidelines available and useful on an international basis. Although the method of administration in this study was relatively homogeneous, a large variety (as demonstrated through a large standard deviation) of concentrations (especially for Bediol) used both at the initiation of treatment and (even more so) at the first follow-up was observed. Furthermore, in Italy, a formidable variety of indications for the prescription of cannabinoids. Several types of chronic pain syndromes have been treated. In order to obtain more reproducible data, in the near future, the authors intend to better focalize indications for specific pain syndromes, such as intractable neuropathic pain. That a low rate (22\%) of discontinuation of cannabinoids (certainly compared to discontinuation rates of opioid analgesics) was observed is clearly encouraging, particularly given that the iatrogeneses of cannabinoids in pain treatment are likely less substantial than those of opioids.

This study has several important limitations. First, it is a retrospective analysis of only one center, and unfortunately, we are compelled to acknowledge that this somewhat limits our understanding of the efficacy of medical cannabinoids for chronic pain. Accordingly, we will continue to collect data from the five hospitals other than the Azienda Ospedaliero Universitaria Pisana and intend to publish data that will address inter-facility variance in outcomes. Second, future investigation will analyze the impact of concomitant treatments, which will require a sufficient number of subjects for the performance of analyses of covariance. Thus, we expect to move toward a better understanding of whether cannabinoid products are more effective as a monotherapeutic approach to chronic pain treatment as opposed to a component of multimodal care. Finally, as the vast majority of patients enrolled in the study used the high-THC Bedrocan, we were unable to assess the difference between cannabinoid treatments with different ratios of THC and CBD. Future investigation will certainly look at this issue, as questions regarding the medical benefits and safety of high-THC, lowCBD cannabis have arisen. ${ }^{10}$

\section{Conclusion}

An initial analysis of the Italian clinical practice of the use of cannabinoids for chronic pain syndromes in a reasonably large population is presented. Even with the heterogeneity of the sample size and the limited data available, it can be stated that the treatment seems to be effective and safe in the majority of patients, even though the safety and effectiveness data should be confirmed in a trial better designed to assess them. Nevertheless, additional data from a variety of types of trials are needed in order to better understand the benefits of cannabinoids to chronic pain sufferers. It is important for the Italian and other European pain societies to more thoroughly investigate this topic in order to provide clearer and more useful guidelines, which will more adequately guide physicians in the use of this drug in the treatment of chronic pain.

\section{Disclosure}

The authors report no conflicts of interest in this work.

\section{References}

1. Jensen B, Chen J, Furnish T, Wallace M. Medical marijuana and chronic pain: a review of basic science and clinical evidence. Curr Pain Headache Rep. 2015;19(10):50.

2. Deshpande A, Mailis-Gagnon A, Zoheiry N, Lakha SF. Efficacy and adverse effects of medical marijuana for chronic noncancer pain: systematic review of randomized controlled trials. Can Fam Physician 2015;61(8):e372-e381.

3. Mehmedic Z, Chandra S, Slade D, et al. Potency trends of D9-THC and other cannabinoids in confiscated cannabis preparations from 1993 to 2008. J Forensic Sci. 2010;55:1209-1217.

4. Pertwee RG. The diverse $\mathrm{CB} 1$ and $\mathrm{CB} 2$ receptor pharmacology of three plant cannabinoids: delta9-tetrahydrocannabinol, cannabidiol and delta9-tetrahydrocannabivarin. Br J Pharmacol. 2008;153(2): 199-215.

5. Ashton H. Pharmacology and effects of cannabis: a brief review. $\mathrm{Br} J$ Psychiatry. 2001;178:101-106.

6. Gaoni Y, Mechoulam R. Isolation, structure, and partial synthesis of an active constituent of hashish. J Am Chem Soc. 1964;86:1646-1647.

7. Demuth D, Molleman A. Cannabinoid signaling. Life Sci. 2006;78(6): 549-563.

8. Campos AC, Moreira FA, Gomes FV, Del Bel EA, Guimarães FS. Multiple mechanisms involved in the large-spectrum therapeutic potential of cannabidiol in psychiatric disorders. Philos Trans $R$ Soc Lond B Biol Sci. 2012;367:3364-3378.

9. Iversen L. The Science of Marijuana. 2nd ed. Oxford: Oxford University Press; 2008.

10. Schatman ME. Medical marijuana: the state of the science. Medscape. 2015 Feb 6. 
11. Koppel BS, Brust JC, Fife T, et al. Systematic review: efficacy and safety of medical marijuana in selected neurologic disorders: report of the Guideline Development Subcommittee of the American Academy of Neurology. Neurology. 2014;82:1556-1563.

12. Borodovsky JT, Crosier BS, Lee DC, Sargent JD, Budney AJ. Smoking, vaping, eating: is legalization impacting the way people use cannabis? Int J Drug Policy. 2016;36:141-147.

13. Hill KP. Medical marijuana for treatment of chronic pain and other medical and psychiatric problems. JAMA. 2015;313(24):2474-2483.

14. Andreae MH, Carter GM, Shaparin N, et al. Inhaled cannabis for chronic neuropathic pain: a meta-analysis of individual patient data. J Pain. 2015; 16(12):1221-1232.

15. Aggarwal SK. Cannabinergic pain medicine: a concise clinical primer and survey of randomized-controlled trial results. Clin J Pain. 2013;29:162-171.
16. Ware MA, Wang T, Shapiro S, Collet JP; COMPASS Study Team. Cannabis for the Management of Pain: Assessment of Safety Study (COMPASS). J Pain. 2015;16(12):1233-1242.

17. Savage SR, Romero-Sandoval A, Schatman M, et al. Cannabis in pain treatment: clinical and research considerations. J Pain. 2016;17(6):654-668.

18. Choo EK, Feldstein Ewing SW, Lovejoy TI. Opioids out, cannabis in: negotiating the unknowns in patient care for chronic pain. JAMA. 2016; 316(17): 1763-1764.

19. Law: Decreto 9 novembre 2015. GU Serie Generale n.279 del 30-11-2015.

20. Bedrocan: medical cannabis varieties. Available from: http://www. bedrocan.nl/english/products.html. Accessed January 8, 2017.

21. Von Elm E, Altman DG, Egger M, et al; STROBE Initiative. Strengthening the Reporting of Observational Studies in Epidemiology (STROBE) statement: guidelines for reporting observational studies. BMJ. 2007;335(7624):806-808.
Journal of Pain Research

\section{Publish your work in this journal}

The Journal of Pain Research is an international, peer reviewed, open access, online journal that welcomes laboratory and clinical findings in the fields of pain research and the prevention and management of pain. Original research, reviews, symposium reports, hypothesis formation and commentaries are all considered for publication.
Dovepress

The manuscript management system is completely online and includes a very quick and fair peer-review system, which is all easy to use. Visit http://www.dovepress.com/testimonials.php to read real quotes from published authors. 